\title{
Estrategias lúdicas interdisciplinarias para fortalecer una cultura ambiental en estudiantes de básica secundaria ${ }^{1}$
}

\section{$\overline{\text { Interdisciplinary ludic strategies to strengthen an }}$ environmental culture in secondary school students}

DOI: http://dx.doi.org/10.17981/cultedusoc.9.3.2018.47

Artículo de investigación. Fecha de recepción: 15/06/2018. Fecha de aceptación: 27/11/2018

Raisa Arroyo Fontalvo';

Atilio Zambrano Ojeda; Nestor García Cabarcas;

María Escorcia Sarmiento y Sofanor Borja De La Rosa ${ }^{3}$

Institución educativa Departamental Baldomero Sanín Cano de San Rafael-Remolino (Colombia) institucionsanrafaelremolino@gmail.com

Para citar este artículo:

Arroyo, R., Zambrano, A., García, N., Escorcia, M. y Borja, S. (2018). Estrategias lúdicas interdisciplinarias para fortalecer una cultura ambiental en estudiantes de básica secundaria. Cultura. Educación y Sociedad 9(3), 407-412. DOI: http://dx.doi.org/10.17981/ cultedusoc.9.3.2018.47

\section{Resumen}

El proceso investigativo busca fortalecer lúdicamente de forma interdisciplinar la cultura ambiental de los estudiantes de básica secundaria. Teniendo como principal objetivo determinar la incidencia de las estrategias lúdicas interdisciplinarias en la consolidación de cultura ambiental en la Institución Educativa Departamental Baldomero Sanín Cano. El estudio investigativo se apoya oportunamente en un tipo de investigación mixta, aplicándose los siguientes instrumentos de recolección de información: sondeos, entrevistas, encuestas a estudiantes, docentes y padres de familia, análisis de variables cuantitativas y cualitativas. Los resultados evidenciaron diversas problemáticas ambientales que requieren prontamente intervención. A través del proceso investigativo y dando respuesta al objetivo general planteado en la investigación, se insta a emplear la educación ambiental desde las escuelas para crear conciencia ambiental en pro de disminuir las conductas ambientales de riesgo que afectan el bienestar de la comunidad en general.

Palabras clave: Cultura ambiental, educación ambiental, estrategias lúdicas, interdisciplinariedad.

\section{Abstract}

The research process seeks to interdisciplinaryly strengthen the environmental culture of secondary school students. The main objective was to determine the incidence of interdisciplinary play strategies in the consolidation of environmental culture in the Departmental Educational Institution Baldomero Sanín Cano. The research study is supported in a timely manner in a type of mixed research, applying the following instruments of information collection: surveys, interviews, surveys to students, teachers and parents, analysis of quantitative and qualitative variables. The results evidenced several environmental problems that require prompt intervention. Through the investigative process and responding to the general objective set out in the research, environmental education is encouraged from schools to create environmental awareness in favor of reducing risky environmental behaviors that affect the well-being of the community in general.

Keywords: Environmental culture, environmental education, play strategies, interdisciplinarity.

1 Este artículo ha sido derivado del Programa de Fortalecimiento de la Cultura Ciudadana y Democrática CT+I a través de la IEP apoyada en TIC en el Departamento de Magdalena: CICLON

2 Líder del grupo de investigación: "Vigías Del Ambiente".

3 Docentes de la institución educativa Departamental Baldomero Sanín Cano de San Rafael-Remolino, del grupo de investigación:

- The author; licensee Universidad de la Costa - CUC.

Cultura, Educación y Sociedad vol. 9 no. 3, pp. 407-412. Diciembre, 2018

Barranquilla. ISSN 2389-7724 Online 


\section{Introducción}

La educación en el contexto actual es una manifestación humana que se establece como el resultado de las diversas interacciones entre el ser humano y su espacio sociocultural. Con el tiempo, los sistemas educativos se han ajustado a las problemáticas y cambios históricos, exponiendo los métodos más propicios para materializarlos en un momento específico. Se busca entonces, acciones que impulsen un sentido crítico y creativo para la solución de problemas complejos desde una perspectiva integral, concreta y real (Maquilón, 2011). La educación es un proceso de construcción de conocimientos y significados para el estudiante, donde él es el eje central del proceso y participa activamente desde la planeación misma, de actividades de aprendizaje y cuyo fin es que cada estudiante alcance su etapa superior de desarrollo (Flórez, 2005).

El ser humano ha percibido el deterioro a la naturaleza resultado de las relaciones desequilibradas entre este y su medio ambiente. Se ha considerado inaplazable el papel de la educación formal en lo referente a la formación de valores y comportamientos positivos frente a la biósfera, situando a la niñez y a los adolescentes a la delantera de la salvaguardia de los recursos naturales. Al respecto Morín (2000) indica que en el futuro la educación deberá ser basada en una enseñanza universal y que se centre en la condición humana.

En este sentido, es importante perfilar una seria transformación en las formas de relaciones permanentes que sostienen los seres humanos con su entorno natural inmediato, a través de la expansión de un proceso educativo que apropie a sus participantes no sólo en acciones puntuales (e.g. siembra de árboles, reciclaje de residuos o la adecuada utilización de las diferentes herramientas tecnológicas) sino que también apunte directamente a la construcción de un nuevo y sólido esquema de valores y conciencia de los problemas ambientales, la aplicación de correctivos y de prácticas armónicas con la naturaleza.

Atendiendo a lo anterior, Novo (1999) expresa que la educación ambiental es el epicentro donde confluyen diferentes perspectivas de análisis de la realidad ambiental y social que propenden por modificar sustancialmente el sistema preponderante en la contemporaneidad de relaciones reciprocas entre ambas, para integrar nuevos criterios que no provoquen tantas alteraciones.

Con este proceso, se hace firme la necesidad de implantar acciones hacia un mejor bienestar y calidad de vida a nivel individual y progresivamente hacia lo colectivo; se trata a decir de autores como Carrera, Bravo, Marín y Crasto (2013) la búsqueda de estrategias que promuevan la integración entre los diferentes actores del sistema social, incluido los de carácter educativo; de esta manera, la educación ambiental potencializa la estructuración cognoscitiva de los diferentes saberes, partiendo de su articulación a los problemas sociales y ambientales, expande la confluencia de los valores como puente de mediación entre lo social, lo cultural y lo ambiental y aúna esfuerzos para que en todos los contextos sociales a nivel global, nacional y local se sienten los cimientos para estimular el desarrollo sostenible como el camino más certero hacia la supervivencia de nuestra especie y las de las diferentes formas de vida que habitan el planeta.

Se debe asumir la educación ambiental como un enfoque integrado e interdisciplinar en el currículo a fin de sentar bases para el desarrollo sostenible, 
basados en proyectos de acción conjunta; pero en la mayoría de las instituciones colombianas se reduce solo a la asignatura de medio ambiente, por lo que hay deficiencias en cuanto a la inclusión de los contenidos medio ambientales en la estructura curricular (Ospina y Rave, 2015; Rojas, 2009).

La educación lúdica busca “...la estimulación de las relaciones cognoscitivas, afectivas, verbales, psicomotoras, sociales, la mediación socializadora del conocimiento y la provocación de una reacción activa, crítica creativa..." (Nunes de Almeida, 1994) de los estudiantes, a fin de exponer a estos a temáticas y problemas del entorno, y despertar en ellos su capacidad creativa y productiva para la solución de problemas. A través de la lúdica se puede incrementar el interés en niños y jóvenes por explorar y descubrir el entorno, además, se estimula la participación en equipos, la cooperación, el respeto, la creatividad y permite un aprendizaje significativo, en este caso el niño toma conciencia ecológica al interactuar con la naturaleza y palpar sus problemáticas.

La preocupación central es ¿Cómo lograr un cambio de actitud frente al comportamiento humano y su forma de ver todo lo que le rodea? Para que, el hombre se reconozca como parte de la naturaleza y fomente el respeto por esta, de manera que se pueda encontrar un lugar de acción desde la escuela, es aquí donde el proceso educativo formal y a partir de la práctica pedagógica puede problematizar comportamientos y actitudes. En efecto, es desde la escuela y la práctica de una pedagogía del medio ambiente que es posible lograr una ética del respeto a la naturaleza y una educación ambiental para lograr un cambio en el actuar del ser humano. Desde la Institución Educativa Departamental Baldomero Sanín Cano, se planteó la actividad lúdica como fortalecimiento de la educación ambiental en los estudiantes de bachillerato.

\section{Metodología}

El trabajo de investigación "estrategias lúdicas interdisciplinarias para el fortalecimiento de una cultura ambiental" se llevó a cabo en la Institución Educativa Departamental Baldomero Sanín Cano, ubicado en el corregimiento de San Rafael, Municipio de Remolino, Departamento del Magdalena, institución oficial y de carácter mixto.

El presente proyecto investigativo está sujeto dentro de una investigación mixta, la cual incluye una sucesión de procesos sistematizados, empíricos y críticos, en su desarrollo utiliza la recolección y análisis de variables cuantitativas y cualitativas. Se enmarca en el paradigma crítico social, ya que se ocupa del diagnóstico de un problema en un contexto específico, basadas en las realidades cotidianas generadas por actores en su relación con el mundo natural en el cual viven.

Para la realización del proyecto se definieron cinco fases investigativas desde el diagnóstico a la evaluación (tabla 1). Finalmente se examinó la información recolectada en encuestas, actividades y evaluación final para determinar si se alcanzaron los objetivos propuestos. En este punto se tuvo en cuenta la estimación y cualificación de los conocimientos, actitudes, aptitudes, habilidades de los estudiantes; al terminar cada actividad se efectúa una reflexión conjunta destacando el grado de conocimiento, el reflejo de la conciencia y la sensibilización que demuestra el educando con sus fortalezas y debilidades.

Para colectar la información se implementaron diversas técnicas como se resumen en la tabla 2. 
TABLA 1

Fases de investigación implementadas.

\begin{tabular}{|c|c|c|}
\hline Fase & Actividad & Descripción \\
\hline Fase 1 & Diagnóstico & $\begin{array}{l}\text { Evaluación problemática ambiental en la escuela. Encuestas a } \\
\text { estudiantes, docentes y padres de familia. }\end{array}$ \\
\hline Fase 2 & Análisis & $\begin{array}{l}\text { Interpretación de encuestas, reconocer prioridades y definir } \\
\text { actividades para mejorar el entorno natural. }\end{array}$ \\
\hline Fase 3 & Planeación & $\begin{array}{l}\text { Consultas referentes teóricos. Diseño propuesta pedagógica, basada } \\
\text { en estrategias lúdicas interdisciplinarias. }\end{array}$ \\
\hline Fase 4 & Implementación & $\begin{array}{l}\text { Desarrollo de actividades lúdicas que apuntan a fortalecer una } \\
\text { cultura ambiental en estudiantes de básica secundaria. }\end{array}$ \\
\hline Fase 5 & Evaluación & $\begin{array}{l}\text { Cuestionario y prueba de Likert para valorar las estrategias y } \\
\text { eficiencia en el desarrollo de la cultura ambiental. Evaluación final de } \\
\text { objetivos. }\end{array}$ \\
\hline
\end{tabular}

TABLA 2

Técnicas e instrumentos de recolección de la información

\begin{tabular}{|c|c|}
\hline Técnica o Instrumento & Objetivo \\
\hline Revisión documental & $\begin{array}{l}\text { Identificar trabajos de relevancia del proceso investigativo que } \\
\text { soportan el cuerpo teórico y análisis de datos (Orozco y Caviedes, } \\
\text { 1996) }\end{array}$ \\
\hline Cuestionarios & $\begin{array}{l}\text { Recolección de datos a través de preguntas predeterminadas (Rojas, } \\
\text { Múnevar y Quintero (2000) }\end{array}$ \\
\hline $\begin{array}{l}\text { Observación } \\
\text { participante }\end{array}$ & $\begin{array}{l}\text { Recolección de datos a través de observadores involucrados en campo, } \\
\text { y analizan la interacción individual y grupal (Rojas y Toro, 1997) }\end{array}$ \\
\hline Test de observación & $\begin{array}{l}\text { Determinar desempeño académico de docentes y estudiantes con } \\
\text { relación a la educación ambiental (Flórez, 1999) }\end{array}$ \\
\hline Matriz de Vester & $\begin{array}{l}\text { Tabla de doble entrada para caracterizar los problemas ambientales } \\
\text { en la institución educativa en causas y consecuencias, además de } \\
\text { evaluar la actitud individual y colectiva de los miembros ante los } \\
\text { mismos (Correa et al. citado por Tobasura y Sepúlveda, 1997) }\end{array}$ \\
\hline Bosque de problemas & $\begin{array}{l}\text { Permite visualización global de la problemática ambiental de la } \\
\text { comunidad educativa. Se plantean tres arboles: ambiente físico, } \\
\text { recursos naturales y problemas socio-ambientales. Las raíces de los } \\
\text { árboles representan problemas activos, los troncos los problemas } \\
\text { críticos y en ramas problemas pasivos o consecuencias (Correa et al. } \\
\text { citado por Tobasura y Sepúlveda, 1997) }\end{array}$ \\
\hline Encuesta & $\begin{array}{l}\text { Información de los sujetos de estudio, proporcionada por ellos mismos, } \\
\text { sobre actitudes, opiniones, sugerencias (Orozco y Caviedes, 1996). } \\
\text { Aplicada a padres de familia }\end{array}$ \\
\hline Prueba Likert & $\begin{array}{l}\text { Escala psicométrica, las respuestas deben estar dadas en un nivel de } \\
\text { acuerdo o desacuerdo respecto a una pregunta en particular o una } \\
\text { declaración. Se utiliza para medir la actitud o predisposición de los } \\
\text { encuestados ante contextos sociales particulares. }\end{array}$ \\
\hline
\end{tabular}




\section{Resultados}

La aplicación del estudio en la Institución Educativa Departamental Baldomero Sanín Cano permitió determinar la alta incidencia que posee la implementación de estrategias lúdicas para lograr consolidar una cultura ambiental acorde a las necesidades de la población. Dada la relevancia que tiene la temática ambiental a nivel general debido a las múltiples implicaciones que ha tenido el cambio climático por las acciones que se han realizado y que han ocasionado daño ambiental. La aplicación de objetivos ha logrado identificar las fortalezas y debilidades con base en las experiencias curriculares que se desarrollan en la institución año tras año y que manejan una temática ambiental, se logró establecer que las temáticas ambientales son de alto interés para los estudiantes pero que la forma de llegar a ellos, suelen ser netamente magistrales y que ellos consideran necesario implementar la lúdica y desarrollar estrategias, para crear un aprendizaje significativo por medio de la apropiación del conocimiento.

Se realizaron actividades lúdicas y recreativas de diferente índole con los estudiantes de básica secundarios de la Institución Educativa Departamental Baldomero Sanín Cano con la finalidad de incentivar la curiosidad y familiaridad de los estudiantes con los temas de investigación acerca de las problemáticas ambientales presentes.

Cabe resaltar que es fundamental la orientación interdisciplinar que se le den a las actividades, teniendo en cuenta el cuidado del medio ambiente en múltiples áreas del conocimiento y que no se trata de un solo tema del área ciencias naturales, con el fin, de crear una cultura ambiental en los estudiantes y que estos a su vez por medio de la implementación del programa de vigías ambientales repliquen en sus comunidades la información a fin de impac- tar la mayor cantidad de personas posibles que cuenten con los conocimientos y herramientas para preservar el ambiente, mediante estilos de vida de conservación, reutilización, reciclaje y preservación.

\section{Discusión}

A partir de las actividades realizadas se evidenció la problemática de la educación ambiental presentada en la institución a través de la observación y con la aplicación de un test a los estudiantes, encuestas a los padres de familia y estudiantes. Además, se logró diseñar partiendo de estos resultados, diversas actividades enfocadas al mejoramiento de la cultura del cuidado ambiental y la construcción o reconstrucción del conocimiento de los estudiantes sobre el medio ambiente (Cortés, 2011; CrissienBorrero, Cortés-Peña \& Herrera-Mendoza, 2016).

Posteriormente se consultó distintos referentes teóricos, así como distintos autores que han hecho aportes significativos a la temática desarrollada, asimismo, se realiza el diseño de la propuesta pedagógica, en el cual se utilizaron distintas estrategias lúdicas apoyadas en la interdisciplinariedad teniendo un aporte significativo en las actividades y resultado de estas fortaleciendo una cultura ambiental en los estudiantes de básica secundaria.

Finalmente se examina la información recolectada en encuestas y actividades para determinar si se alcanzaron los objetivos propuestos. En este punto se tiene en cuenta la estimación y cualificación de los conocimientos, actitudes, aptitudes y habilidades de los estudiantes.

\section{Referencias}

Cortés, O. (2011). Comportamiento proambiental y pensamiento económico en la construcción del desarrollo sostenible. Cultura Educación y Sociedad, 2(1), 4356. 
Carrera, M,. Bravo, O., Marín, F. y Crasto, C., (2013). Futuribles y futurables para el desarrollo sostenible. Líder 22(15), 3974

Crissien-Borrero, T., Cortés-Peña, O. y Herrera-Mendoza, K. (2016). Pro-Environmental Assessment and Sustainable Consumption of Household Public Services in Barranquilla Colombia. The European Proceedings of Social \& Behavioural Sciences-EpSBS, 17, 434-440. http:// dx.doi.org/10.15405/epsbs.2016.11

Flórez, R. (2005). Pedagogía del Conocimiento (2 Ed.). Bogotá, D.C.: Mc Graw Hill.

Flórez, R. (1999). Evaluación Pedagógica y Cognición. Bogotá, D.C.: Mc- Graw Hill.

Landinez-Lamadrid, D., Ramirez-Ríos, D. G., Neira Rodado, D., Parra Negrete, K., y Combita, J. (2017). Shapley Value: its algorithms and application to supply chains. INGE CUC, 13(1), 61-69. https://doi. org/10.17981/ingecuc.13.1.2017.06

Maquilón, J. (2011). La formación del profesorado en el siglo XXI. Propuestas ante los cambios económicos, sociales y culturales. ( $1^{a}$ Ed.). Murcia: Edi.tum Ediciones de la universidad de Murcia.

Morin, E. (2000). El paradigma perdido. Ensayo de bioantropología ( $6^{\mathrm{a}}$ ed.) Barcelona: Kairós.

Novo. (1999). Los desafíos ambientales: reflexiones $y$ propuestas para un futuro sostenible. Madrid: UNESCO-Universitas.
Nunes de Almeida P (1994). Educación Lúdica. Bogotá, D.C.: Ediciones San Pablo.

Ospina, M., y Rave, L. (2015). El PRAE: Una estrategia para la formación ambiental y el fortalecimiento de la identidad territorial en la comunidad de la Institución Educativa La Pintada. [Tesis de posgrado]. Recuperado de http:// bibliotecadigital.udea.edu.co/bitstream/10495/5256/1/martadarleyospina_2016_prae.pdf

Orozco, A. y Caviedes, R. (1996). Elaboración de Proyectos de Investigación. Barranquilla: Universidad del Atlántico.

Rojas, J. (2009). La participación y la interdisciplinariedad como elementos de gestión ambiental- educativa para la construcción de lineamientos curriculares de educación ambiental. Estudio de caso gimnasio Vermont, locadad de Suba. [Tesis de posgrado]. Recuperado de https:/repository.javeriana.edu.co/ bitstream/handle/10554/718/eam 37. pdf?sequence $=1$

Rojas, O., Múnevar, R., y Quintero, J. (2000). Práctica Investigativa En Educación Ambiental. Manizales: Universidad de Caldas.

Rojas, O., y Toro., D. (1997). Educación Ambiental. Manizales: Universidad de Caldas.

Tobasura, I. y Sepúlveda, L. (1997). Proyectos Ambientales Escolares. Manizales: Universidad de Caldas. 